\title{
Hochfrequenzdaten aus der Schifffahrt als Indikator für den deutschen Außenhandel
}

Rund $80 \%$ des weltweiten Handelsvolumens bzw. $70 \%$ des Werts aller gehandelten Güter werden auf dem Seeweg befördert (UNCTAD, 2018). Hochfrequente Schiffspositionsdaten, mit denen sich Bewegungen aller Frachtschiffe weltweit verfolgen lassen, können daher zeitnah verfügbare Informationen zur internationalen Handelsaktivität liefern. Diese Daten werden mittels des Automatischen Identifikationssystems (AIS) durch Radiosignale erhoben und wurden bereits in mehreren Studien, unter anderem des Internationalen Währungsfonds, zur Analyse der Handelsaktivität genutzt (Arslanalp et al., 2019; Cerdeiro et al., 2020). In einer neuen Studie des IfW Kiel macht Stamer (2021) die Schiffspositionsdaten für die Analyse internationaler Warenströme nutzbar. Dafür werden wöchentlich aktualisierte Positionsdaten für alle Containerschiffe weltweit beginnend mit dem Jahr 2015 ausgewertet. Der Datenanbieter FleetMon stellt tägliche Positionen der Frachtschiffe auf See sowie Portcalls, also die Ankünfte und Abfahrten in Häfen, zur Verfügung. Insgesamt handelt es sich um etwa 2 Mio. Positionen und 500.000 Hafenankünfte pro Jahr. Die Positionsdaten beinhalten den aktuellen Tiefgang sowie den Kurs des Schiffes, die Portcalls, den Tiefgang des Schiffes bei Ankunft und Abfahrt. Angaben zur Ladung der Schiffe liegen nicht vor, allerdings kann die Auslastung über den Tiefgang abgeschätzt werden. Aus diesem Datensatz werden etwa 1.200 monatliche Zeitreihen abgeleitet, z.B. die Auslastung von Containerschiffen aus dem Hamburger Hafen.

Aus diesen Zeitreihen werden mit der Methode der Partial Least Squares (PLS) für jede Zielvariable die jeweils wichtigsten erklärenden Variablen ausgewählt. Ergebnis des PLS-Modells ist eine Prognose der monatlichen Zuwachsrate von Import- und Exportzeitreihen basierend ausschließlich auf Schiffspositionsdaten. Diese Prognose kann auch als Indikator interpretiert und flexibel in konventionellen Prognosemodellen gemeinsam mit anderen Frühindikatoren ausgewertet werden. Die folgenden Analysen beschränken sich auf den Nowcast für den deutschen Warenhandel (Kiel Trade Indicator).

(C) Der/die Autor:in(nen) 2021. Open Access: Dieser Artikel wird unter der Creative Commons Namensnennung 4.0 International Lizenz veröffentlicht (creativecommons.org/licenses/by/4.0/deed.de).

Open Access wird durch die ZBW - Leibniz-Informationszentrum Wirtschaft gefördert.
Für den deutschen Außenhandel wählt die PLS-Methode aus dem umfassenden Schiffsdatensatz neben deutschen Häfen auch Positionen in Nord- und Ostsee sowie dem Mittelmeer aus. Weitere Häfen in Europa und anderen Weltregionen gehen in das Modell ein und spiegeln die europäische bzw. globale wirtschaftliche Aktivität wider. Als Zielvariablen werden für diese Analyse die preisbereinigten monatlichen Importe und Exporte in Abgrenzung des Spezialhandels verwendet. Der Kiel Trade Indicator wird zur Monatsmitte und zum Beginn des Folgemonats berechnet. Damit liegt der Indikator bis zu zwei Monate vor der Veröffentlichung der Außenhandelszahlen vor. Um die Berechnung des Indikators in der Praxis zu simulieren, werden vergangene Werte mit Hilfe einer „outof-sample"-Analyse prognostiziert. Für die Modellierung werden demnach jeweils alle Beobachtungen einbezogen mit Ausnahme jener, die geschätzt werden soll. ${ }^{1}$

Die resultierenden Handelsindikatoren weisen einen deutlichen Gleichlauf mit den Zuwachsraten des Spezialhandels auf (vgl. Abbildungen 1 und 2). Dies trifft sowohl auf die Exporte als auch auf die Importe zu, obwohl der Anteil des Seeverkehrs für Deutschland am Gesamtwert des Warenhandels nur rund ein Fünftel beträgt. Maßgeblich hierfür dürfte sein, dass durch die Auswahl der Häfen und Positionen Proxys sowohl für die deutsche wirtschaftliche Aktivität, als auch für die europäische und globale Konjunktur in die Berechnung einfließen.

Auch der Einbruch des Warenhandels im Zuge der ersten Corona-Welle im Frühjahr 2020 wird von den schiffsdatenbasierten Indikatoren mit zunehmender Datenverfügbarkeit nachvollzogen. Vor allem der starke Rückgang im April 2020 wird unter Einbeziehung der Schiffsdaten bis zum Monatsende recht gut prognostiziert, wenngleich er etwas unterschätzt wird. Der Rückgang im März wird dagegen noch nicht durch den Indikator erfasst. Dieser ist vor allem auf den Rückgang der Handelsaktivität auf dem Landweg zurückzuführen. So lagen die nominalen Warenexporte auf dem Seeweg im März 2020 nur rund 4,5\% unter ihrem Vorjahresniveau und erst im Folgemonat brachen sie um knapp ein Drittel ein. Die Einschränkungen des internatio-

1 Aufgrund dieser Methode kann es auch für vergangene Monate zu unterschiedlichen Werten kommen, da sich die Algorithmen eigenständig verbessern. 


\section{Abbildung 1}

Nowcast und ex-post Werte der preisbereinigten monatlichen Exporte

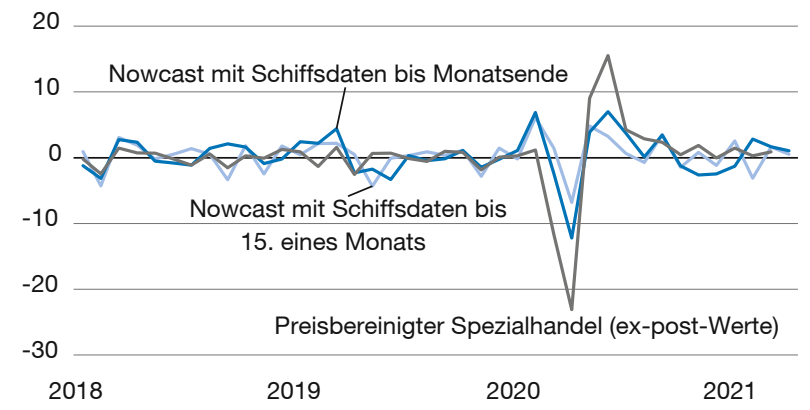

Monatsdaten, letzter Wert: März (Spezialhandel), April (Indikatoren).

Quelle: Destatis, Berechnungen des IfW Kiel.

nalen Warenhandels aufgrund der Lockdown-Maßnahmen zur Eindämmung der Pandemie schlagen sich daher erst im April im Indikator nieder. Dies gilt auch für die Importe. Im Hinblick auf die aktuelle Entwicklung deuten die Handelsindikatoren für die Exporte zum Auftakt des zweiten Quartals 2021 eine Fortsetzung der Erholung an.

Zur Evaluierung der Prognosegüte des Kiel Trade Indicator für die monatlichen deutschen Exporte und Importe werden zum Vergleich Prognosemodelle mit konventionellen Frühindikatoren geschätzt. Dafür werden die ifo Exporterwartungen, das ifo Importklima sowie der RWI/ ISL-Containerumschlag-Index verwendet. Der durchschnittliche Prognosefehler, gemessen mittels des Root Mean Square Error (RMSE), des Kiel Trade Indicator für

\section{Abbildung 3}

Root Mean Square Error verschiedener Prognosemodelle vor und während des Corona-Schocks

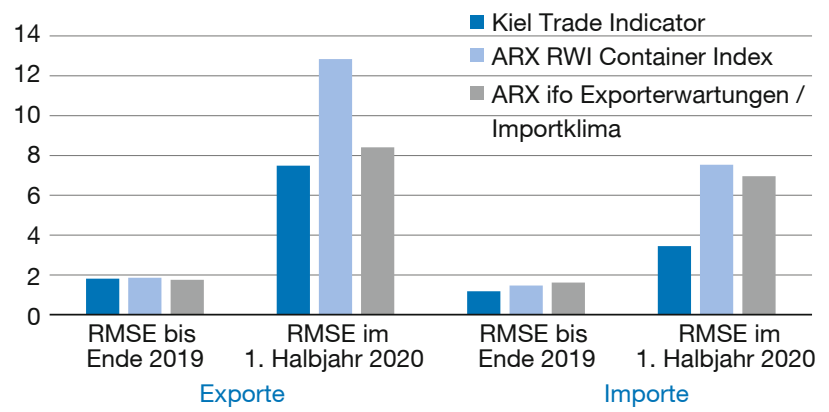

Anmerkungen: Für Prognoseevaluation werden ARX-Modelle mit jeweils einem konventionellen Frühindikator für den deutschen Export geschätzt. Es wird - wie für die Erstellung des Kiel Trade Indicators - die „leave-oneout"-Methodik angewendet. Die Zahl der Lags wird für jede Schätzung neu mittels des AIC-Kriteriums ausgewählt. Die ARX-Modelle mit den konventionellen Frühindikatoren werden jeweils für den gesamten Zeitraum ihrer Verfügbarkeit geschätzt. Für Details zur Methodologie: Stamer (2021).

Quelle: Berechnungen des IfW Kiel.

\section{Abbildung 2}

Nowcast und ex-post Werte der preisbereinigten monatlichen Importe

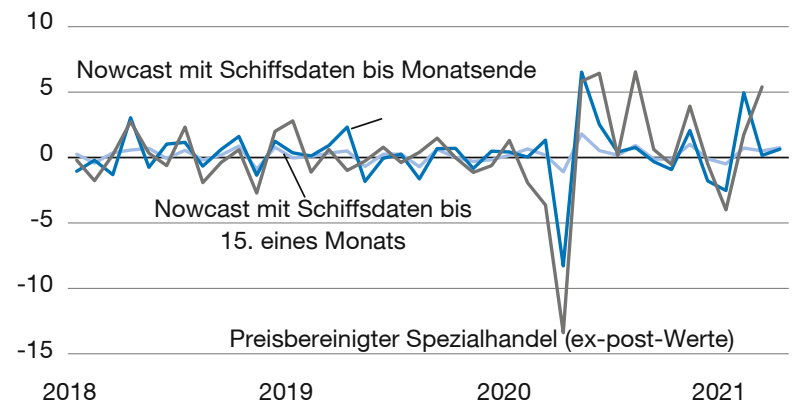

Monatsdaten., letzter Wert: März (Spezialhandel), April (Indikatoren).

Quelle: Destatis, Berechnungen des IfW Kiel.

die monatlichen deutschen Exporte im Zeitraum von 2015 bis 2019 ist vergleichbar mit dem der konventionellen Frühindikatoren; für die Importe liegt der mittlere Prognosefehler des Handelsindikators sogar merklich unter dem anderer Frühindikatoren (vgl. Abbildung 3). Im ersten Halbjahr 2020 - also während der großen, Corona-bedingten Schwankungen im internationalen Handel - schneiden die neuen Indikatoren im Vergleich der mittleren Prognosefehler deutlich besser ab als konventionelle Frühindikatoren. Dies gilt für Exporte und Importe, für letztere jedoch in besonderem Maße.

Die Ergebnisse zeigen, dass der Kiel Trade Indicator für den deutschen Warenhandel eine wertvolle Ergänzung zu konventionellen Frühindikatoren darstellt. Die Indikatoren sind ein Beispiel dafür, wie „Big Data“ für die makroökonomische Analyse eingesetzt werden können (Ademmer et al., 2021). Aufgrund der nahezu tagesaktuellen Verfügbarkeit der Daten ist der Kiel Trade Indicator insbesondere auch in Krisenzeiten, in denen hohe Unsicherheit herrscht und traditionelle Indikatoren oft erst mit größerer Verzögerung Signale liefern, nützlich.

Saskia Meuchelböck, Vincent Stamer Institut für Weltwirtschaft saskia.meuchelboeck@ifw-kiel.de, vincent.stamer@ifw-kiel.de

\section{Literatur}

Ademmer, M. et al. (2021), Big Data in der makroökonomischen Analyse, Kieler Beiträge zur Wirtschaftspolitik, Nr. 32

Arslanalp, S., M. Marini und P. Tumbarello (2019), Big data on vessel traffic: Nowcasting trade flows in real time, IMF Working Paper, Nr. 19/275.

Cerdeiro, D. A., A. Komaromi, Y. Liu und M. Saeed (2020), World seaborne trade in real time: A proof of concept for building ais-based nowcasts from scratch, IMF Working Paper, Nr. 20/57.

Stamer, V. (2021), Thinking outside the container: A machine learning approach to forecasting trade flows, Kiel Working Paper, Nr. 2179.

UNCTAD (2018), Review of Maritime Transport 2018, United Nations. 